\title{
The electromechanical converter in the systems of desulfurisation of crude oil
}

\author{
Denis Kuimov ${ }^{1, *}$, Maxim Minkin ${ }^{1}$ \\ ${ }^{1}$ Don State Technical University, Chair "Production Process Automation”, 344000, Gagarin square, Rostov-on-Don, Russia
}

\begin{abstract}
In article authors have investigated a question of a possibility of application of hydrodynamic cavitation processing of crude oil for the purpose of decrease in content of sulphurous compounds. The electromechanical converter with a secondary discrete part, the being device exciting in the processed material the cavitation and shock field by means of heavy traffic of big set of ferromagnetic elements under the influence of external magnetic field is presented. Features of initiation of hydrodynamic cavitation on the example of the movement of a single ferromagnetic element are considered. Results of the mathematical description of process of desulphurization of crude oil are presented. On the basis of economic efficiency of the presented technological process, authors have offered options of introduction of technology on production objects.
\end{abstract}

\section{Introduction}

The modern oil branch of the Russian Federation is characterized by increase in a share of high-sulphurous grades of the extracted oil in the general structure of reserves of hydrocarbonic raw materials. As of 2016 the share high-sulphurous $(1,68 \%)$ and sulphurous grades $(0,61-1,68 \%)$ has made $69 \%$, and in the future will only increase, taking into account exhaustion of fields of "easy" grades.

Widespread ways of processing of raw materials for the purpose of minimization of content of sulphurous compounds are: hydrotreating, the adsorptive cleaning and sonocatalitic desulphurization. All listed methods have the proved efficiency in oil desulphurization processes, and have already found the application at many oil refineries of various power. Nevertheless, they aren't deprived of shortcomings among which: high power consumption, irrevocable losses of expensive catalysts, complexity of system.

According to many scientists both foreign, and domestic, a perspective method of processing of oil for the purpose of change of rheological properties and change of fractional structure is hydrodynamic cavitation processing.

Within the previous researches [1,2], authors of work have studied the phenomenon of hydrodynamic cavitation in devices with a secondary discrete part, the new configuration of the electromechanical converter is offered and the possibility of its application in the systems of primary oil refining for the purpose of decrease in content of sulphurous compounds is investigated.

The purpose of this research is:
1. Theoretical assessment of overall performance of the device;

2. Economic assessment of the offered technology.

\section{Main part}

\subsection{Technological process}

The electromechanical transformer with a secondary discrete part represents the cylindrical multiphase inductor with the concentrated winding, on the place of a rotor the working camera from non-magnetic material is located. As a working organ the ferromagnetic elements located in the working camera and making under the influence of an outside magnetic field heavy traffic from one active pole to another are applied. Movement of ferromagnetic elements with high speeds generates hydrodynamic cavitation in processed liquid material.

The phenomenon of hydrodynamic cavitation $[3,4,5]$ arises at the time of approximation of liquid pressure to pressure of saturated steam of liquid raw materials. In case of the close values in liquid processed material abundant separation of the bubbles filled with gas is watched. The considerable effect of cavitation processes is connected to high concentration of the energy emitted in the course of collapse in the processed environment thereby initiating processes of corrupting of sulphurous compounds. This phenomenon at the time of achievement by a bubble of the minimum radius preceding collapse is explained by the small volume of substance.

In case of collapse of a cavitation bubble kinetic energy [6,7] which turns into activation energy of different responses is emitted:

\footnotetext{
* Corresponding author: muzhik1010@yandex.ru
} 


$$
W_{\text {cin }}=2 \pi \rho U^{2} R^{4} \int_{R} \frac{d r}{r^{2}}=2 \pi \rho U^{2} R^{3}
$$

where, $U$ - the speed of a spherical wall; $R$ - instant radius of the slamming sphere.

Speed of a spherical wall is determined by the following formula:

$$
U=\sqrt{\frac{2}{3} \frac{P}{\rho}\left(\frac{R_{0}^{3}}{R^{3}}-1\right)},
$$

where, $R_{0}$ - the initial radius of a bubble.

We will write down the total energy emitted at collapse of a set of cavitation cavities through number of cavitation cavities on a formula:

$$
N=k_{c} \cdot V_{l}
$$

where, $k_{c}$ - concentration of cavitation cavities which for oil accepts value $10^{11} 1 / \mathrm{m}^{3}, V_{l}$ - liquid volume with the developed cavitation, $\mathrm{m}^{3}$.

The way passed by a single ferromagnetic element on the studied trajectory will be accepted to liquid volume with the developed cavitation.

Considering expressions (1-3), we will write down a formula of the energy which is marked out at collapse of cavities at supercavitation:

$$
W_{\text {cin }}=\frac{4}{3} k_{c} V_{l} \pi P\left(\frac{R_{0}^{3}}{R^{3}}-1\right) R^{3},
$$

Having considered effect of cavitation and the energy emitted at collapse of cavitation cavities, further for a theoretical research of efficiency of work of the electromechanical converter with a secondary discrete part it is necessary to investigate fractional composition of the processed raw materials.

The analysis of fractional composition of liquid hydrocarbons of various fields has shown that distribution of sulfur has difficult character. For a theoretical research sulphidic oil of the Arlansky field has been chosen. For the purpose of simplification of calculations, all variety of sulphurous compounds has been divided into three groups with the closest molar mass and energy of communication (tab. 1).

Table 1. Sulphurous compounds

\begin{tabular}{|l|l|l|l|l|}
\hline № & Name & $\begin{array}{l}\text { Share from } \\
\text { the general } \\
\text { contents } \\
\text { CC, \% }\end{array}$ & $\begin{array}{l}\text { Average } \\
\text { molar } \\
\text { weight, } \\
\mathrm{g} / \mathrm{mol}\end{array}$ & $\begin{array}{l}\text { Average } \\
\text { energy of a } \\
\text { rupture of } \\
\text { communic } \\
\text { ation, } \\
\mathrm{kJ} / \mathrm{mol}\end{array}$ \\
\hline 1 & Thiophene & 76,17 & 44 & 82 \\
\hline 2 & Sulphide & 23,6 & 50 & 80 \\
\hline 3 & Mercaptan & 0,23 & 80 & 360 \\
\hline
\end{tabular}

Within model of process of desulfurization it is supposed that reaction of a rupture of sulphurous compounds is carried out in one stage on C-S communication, at achievement by cavitation of necessary value of energy of a rupture of communication, in the set volume, at the same time calculation was carried out in two steps. At the first stage the volume of the liquid hydrocarbonic raw materials subjected to processing as a result of hydrodynamic cavitation paid off. The way passed by ferromagnetic elements with a speed called further critical at which the condition of emergence of the supercavitation field is satisfied was accepted to volume.

$$
V=N_{e l} \pi r^{2} L_{\text {mean }} 15 k_{t r}+N_{e l} \pi r^{2} L_{c} 15 k_{t r}
$$

where $N_{e l}$ - quantity of the ferromagnetic working elements participating in processing of liquid hydrocarbonic raw materials, piece; $r$ - radius of a single ferromagnetic element in the widest part (a supercavitating profile), $\mathrm{m} ; L_{\text {mean }}$ - average length of a run of an element from one pole to another, $\mathrm{m} ; k_{t r}-\mathrm{a}$ share of the way passed with cavitation.

The value 15, determines the number of switching for one motion cycle of ferromagnetic elements by a circle along boring of the inductor and is equal to quantity of phases of the inductor. In the second item, the value 2 defines the number of switching for which ferromagnetic elements pass average distance on a path lengthways center of the working camera.

The analysis [8] of movement of a one ferromagnetic element, showed that ferromagnetic elements, in case of relocation from one active pole to another, pass through stages of acceleration and braking. As a result, the analysis of process of movement of a one ferromagnetic element, allowed to obtain some averaged data showing a share of the way passed by such element with a speed below the critical, expressed by coefficient path $k_{t r}$ and equal 0,86 .

The amount of the energy emitted in the course of collapse of cavitation cavities is defined with expressions (4) and (5).

At the second stage computation of the molar mass of the sulphurous compounds subjected to cavitation processing was carried out. The amount of substance of sulphurous compounds, is calculated in volume of the liquid hydrocarbonic raw materials subjected to cavitation processing taking into account initial percentage and average molar mass for each group of the compounds provided to tab. 1. on the following expression:

$$
v=m / M
$$

where, $\mathrm{m}$ - a lot of sulphurous compounds; $\mathrm{M}$ - average molar mass of group of sulphurous compounds, $\mathrm{g} / \mathrm{mol}$. Taking into account told the algorithm of calculation of number of the broken-off sulphurous compounds in the course of cavitation processing shown on rice is offered. In fig. 2 the dependence of the broken-off sulphurous compounds on processing time in the electromechanical converter with a secondary discrete part is presented. It is possible to note good removal of sulphurous compounds in the first $20 \mathrm{sec}$. of processing. Further removal is slowed down in view of continuous hashing and decrease in concentration of sulphurous compounds. 
Results of theoretical researches have shown efficiency of the choice as the dominating power influence hydrodynamic cavitation, unlike earlier applied energy of impact, allows to realize effective processing of liquid materials, including oil.

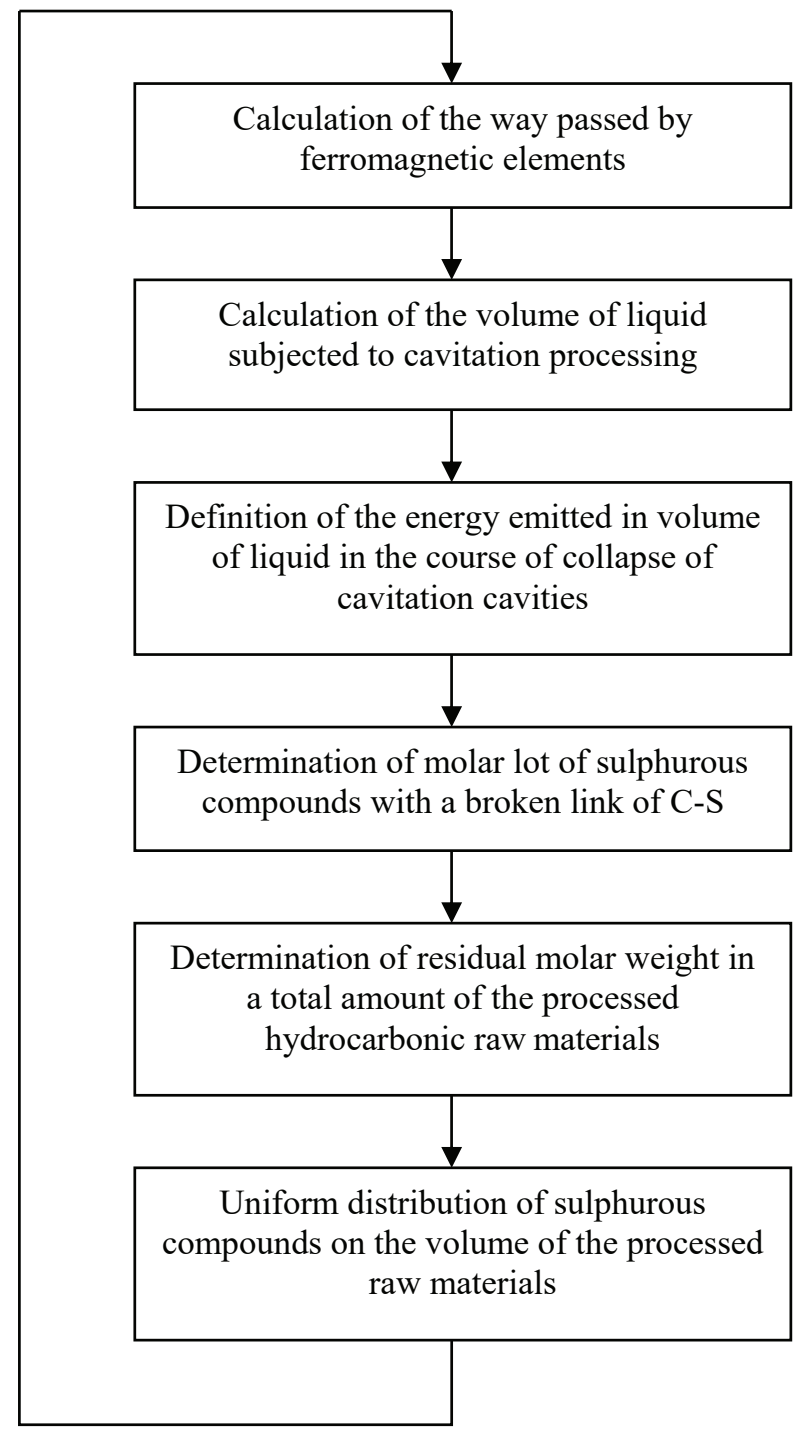

Fig. 1. Algorithm of Calculation of Number of the Broken-off Sulphurous compounds in the course of cavitation processing.

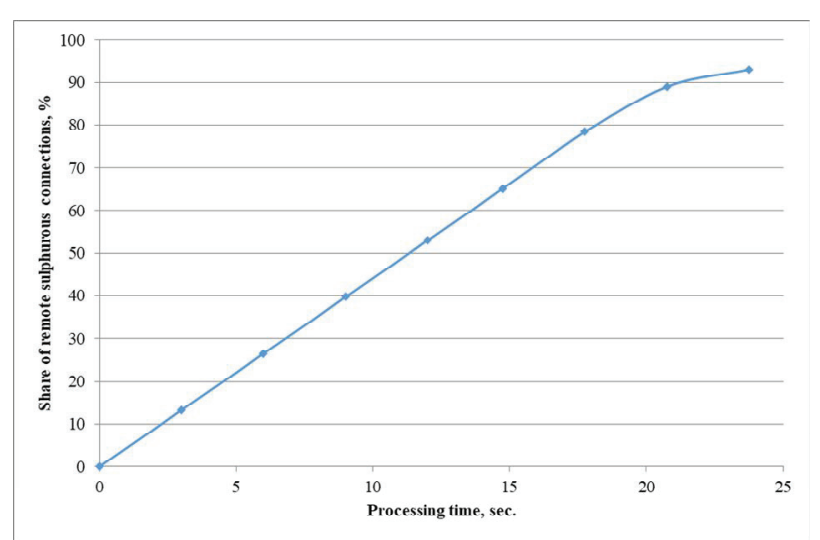

Fig. 2. Desulfurization process the electromechanical converter with a secondary discrete part
Nevertheless, the efficiency and expediency of use of this or that device has to be estimated and compared to the technologies and methods which are the most extended today not only from the point of view of efficiency, but also profitability.

The economic appeal of process of desulfurization of crude oil is connected by the electromechanical converter with a secondary discrete part, first, with lack of need of use of expensive reagents, secondly, with soft conditions of carrying out, moderate temperature and pressure, thirdly, with simplicity of processing equipment.

\section{Assessment of economic efficiency}

Relying on results of a theoretical research of process of desulfurization by the electromechanical converter with a secondary discrete part, approximate calculation of cost of processing of 1 ton of initial raw materials has been carried out with a productivity of system 10t/day and also the comparative analysis with methods of desulfurization of crude oil most widespread and perspective today is carried out.

Certainly, within economic assessment of the presented earlier technological process, the essential value has such concept as an electric power expense as any mechanical or electromechanical device demands her consumption. For this reason accounting of these expenses is essential when accounting expenses on operation of technical devices within the offered technological process.

The lack of need of use of reagents and catalysts reduces the main expenses of production to ensuring energy for implementation of process of movement of a secondary discrete part on the set trajectory.

Thus, it is possible to conclude that the sum of all material inputs on the electric power necessary for processing of 1 ton of crude oil, was respectively, 1078.82 roubles.

Table 2. Costs of the electric power

\begin{tabular}{|c|c|c|c|c|}
\hline $\begin{array}{l}\text { Name of the } \\
\text { equipment }\end{array}$ & $\begin{array}{l}\text { Power of } \\
\text { the } \\
\text { equipme } \\
\text { nt, } \quad \mathrm{kW}\end{array}$ & $\begin{array}{l}\text { The } \\
\text { required } \\
\text { time for } \\
\text { processi } \\
\text { ng of } 1 \\
\text { ton of } \\
\text { initial } \\
\text { raw } \\
\text { materials } \\
\text {, hour }\end{array}$ & $\begin{array}{l}\text { Cost is } \\
\text { one } \\
\mathrm{kW} / \text { ho } \\
\text { ur of } \\
\text { the } \\
\text { electric } \\
\text { power, } \\
\text { rub. }\end{array}$ & $\begin{array}{l}\text { Total } \\
\text { materi } \\
\text { al } \\
\text { inputs, } \\
\text { rub. }\end{array}$ \\
\hline $\begin{array}{l}\text { Electromechani } \\
\text { cal converter }\end{array}$ & 4,9 & 2,45 & 1,16 & 55,7 \\
\hline Capacitor bank & 90 & 2,45 & 1,16 & $\begin{array}{l}1023,1 \\
2\end{array}$ \\
\hline \multicolumn{4}{|c|}{$\begin{array}{l}\text { General costs of processing of } 1 \text { ton of initial raw } \\
\text { materials, rub }\end{array}$} & $\begin{array}{l}1078,8 \\
2\end{array}$ \\
\hline
\end{tabular}

Process most widespread today removal of undesirable impurity of sulphur and sulphurous compounds is hydrotreating process. It agrees $[9,10]$, the cost of processing of 1 ton of liquid hydrocarbonic raw 
materials by method of hydrotreating is 4398,9 rub. It allows to draw conclusions on economic efficiency of the introduced method of cavitation processing and alleged cost cutting for $75,4 \%$.

\section{Conclusion}

Achievement of $90 \%$ of remote sulphurous compounds allows to speak about efficiency of the offered technology of desulphurization of oil and the accompanying device for realization of technology - the electromechanical converter with a secondary discrete part. Possibilities of the electromechanical converter with a secondary discrete part allow to use it not only to mini-oil refinery, but also at large oil refineries, for the purpose of improvement of quality of output products and decrease in economic expenses. At the same time, It should be noted that on the basis of the presented calculations, use of the presented device in the offered configuration is inexpedient for processing the mercaptan types of oil in which in quantity, dominating or commensurable with sulphides and thiophenes, to contain mercaptans, having high values binding energy. Further researches have to be devoted to removal the mercaptan compounds.

\section{References}

1. D.N. Kuimov, M.S. Minkin, A.D. Lukyanov, Materials Science Forum, 870, 671-676 (2016)

2. M.S. Minkin, D.N. Kuimov, M.N. Kulinich, Dynamics of the technical Collection of Works XII of the International Scientific and Technical Conference systems, 392-396 (2016)

3. E. A. Denisyuk, S.P. Shevelyov, The NGIEI Bulletin, 1(2) (2011)

4. A.V. Stavitskaya, Development of methods of oxidizing modifying oil and products of her processing abstract. Wuchang. step. Cand. Tech.Sci., Moscow (2015)

5. V.A. Koryagin, Szhiganiye vodotoplivnykh emul'siy $i$ snizheniye vrednykh vybrosov (monografiya) (Nedra, SPb., 1995), 304p. [in Russian]

6. O.V. Lavrinenko, E.I. Savina, G.V. Leonov, The Polzunovsky messenger, 59-63 (2007)

7. R. Knepp, J. Daley, F.Hemmit, Cavitation (Mir, Moscow, 1974) [in Russian]

8. D.N. Kuimov, News of higher educational institutions. Electromecanics, 5 (547), 11-15 (2016)

9. E.S. Gridneva, Desulfuration of oil products under the influence of ultrasound: abstract. Wuchang. step. Cand.Tech.Sci., Moscow (2010)

10. A.V. Fkopyan, Oxidizing desulphurization of hydrocarbonic raw materials peroxide hydrogen in the presence of salts of transitional metals: abstract. Wuchang. step. Cand. Chem.Sci., Moscow (2015) 